\title{
Herzinfarkt bei jüngeren Frauen häufiger asymptomatisch und tödlich
}

Canto JG et al. Association of age and sex with myocardial infarction symptom presentation and in-hospital mortality. JAMA 2012;307: 813-822.

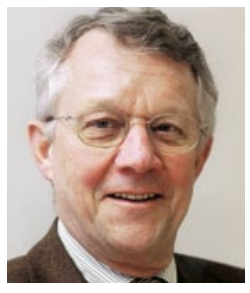

Prof. Dr. med. Heinrich Holzgreve
Fragestellung und Methodik: Die Autoren überprüften den Zusammenhang zwischen dem Geschlecht, den Symptomen bei akutem Herzinfarkt und der Mortalität. Dabei stützten sie sich auf das National Registry of Myocardial Infarction der USA, in dem von 1994 bis 2006 die Daten von 2160671 Patienten mit akutem Herzinfarkt aus 1977 Kliniken dokumentiert wurden.

Ergebnisse: Bei Frauen tritt ein akuter Myokardinfarkt häufiger ohne typische Angina-pectoris-Symptome oder dessen Äquivalente, z.B. Arm-, Hals- oder Kieferschmerzen, auf als bei Männern (42,0 vs. 30,7\%). Dabei besteht eine deutliche Altersabhängigkeit: Die Symptome fehlen umso häufiger, je jünger die Frauen sind. Bei einem Infarkt ohne Brustschmerzen ist die Mortalität bei Frauen höher als bei gleichaltrigen Männern (14,6 vs. $10,3 \%)$. Diese Beobachtung gilt vor allem für jüngere Frauen unterhalb des 55. Lebensjahrs ( $<45$ Jahren: 1,18-faches Risiko; 45-54 Jahre: 1,13-faches Risiko). Mit dem Alter nehmen diese Unterschiede ab und kehren sich sogar um. Alle Veränderungen waren signifikant $(\mathrm{p}<0,001)$.

Schlussfolgerung: Frauen unter 55 Jahren haben bei einem Myokardinfarkt seltener Brustschmerzen und eine höhere Mortalität als Männer.
Kommentar: Die Ursachen für die Geschlechtsunterschiede hinsichtlich der Symptomatik und dem Mortalitätsrisiko sind letztlich unklar, während die Konsequenzen offensichtlich sind: Patienten ohne Brustschmerzen suchen später den Arzt auf und werden wahrscheinlich weniger aggressiv behandelt. Die Autoren fordern, dass diese Unterschiede genauer untersucht und bei der Behandlung berücksichtigt werden.

Prof. Dr. med. Heinrich Holzgreve

Internist, Kardiologische Praxis

Burgstr. 7, D-80331 München

h.holzgreve@t-online.de
Ruusunen A, Voutilainen S, Karhunen Let al. How does lifestyle intervention affect depressive symptoms? Results from the Finnish Diabetes

Prevention Study.

Diabet Med.

2012 Feb 7. doi:

$10.1111 / j .1464$ 5491.2012.03602.x

\section{Diabetes Prevention Study}

\section{Weniger Depressionen nach Änderung des Lebensstils}

Fragestellung: Welchen Einfluss hat eine nachhaltige Lebensstiländerung auf depressive Symptome?

Hintergrund: Das Diabetesrisiko ist zum großen Teil über verhaltensbasierte Risikofaktoren zu erklären. In diesem Kontext kann eine depressive Stimmungslage oder Depression eine relevante Rolle spielen. Über die Hälfte der Typ-2-Diabetiker haben eine Depression. Vorstadien des Diabetes wie Insulinresistenz sind ebenfalls direkt mit einer Zunahme von depressiven Symptomen assoziiert.

Patienten und Methodik: In einer dreijährigen randomisierten klinischen Studie, die als Ergebniskriterium die Prävention des Diabetes beinhaltete, wurden 522 übergewichtige Probanden mit gestörter Glukosetoleranz in eine Interventionsgruppe und eine Kontrollgruppe randomisiert. Die Interventionsgruppe erhielt eine kontinuierliche und regelmäßige Beratung bezüglich ihres Lebensstils. Depressive Symptome wurden mit dem Beck Depression Inventory bei 140 Teilnehmern erhoben. Die Untersuchung war Bestandteil der bekannten finnischen Diabetes Prevention Study, welche die europäische Landmark-Studie in der Diabetesprävention ist.

Ergebnisse: Am Anfang der Studie hatten beide Gruppen vergleichbare Depressionsscores (6,8 und 6,7). Während der Intervention reduzierte sich der Depressionsscore in der Interventionsgruppe um 0,9 und damit 